\title{
Oral administration of Allium sativum extract protects against infectious bursal disease in chickens
}

\author{
Sufen ZHAO, Yuanyuan JIA, Weiwei ZHANG, Lili WANG, Yunfei MA (凶), Kedao TENG (凶) \\ College of Veterinary Medicine, China Agricultural University, Beijing 100193, China
}

\begin{abstract}
Garlic (Allium sativum, Liliaceae) has been safely used for more than 5000 years, and research on garlic extract is rapidly increasing because of its multiple biological functions. The in vivo effects of oral administration of garlic mixture (GM, water-soluble extract) on infectious bursal disease virus (IBDV)-infected specific pathogen free male white leghorn chicken were examined through histopathological, immunohistochemical, and Western blot analyses, and enzyme-linked immunosorbent assay. The results confirmed the protective effects of oral administration of $5 \mathrm{mg} \cdot \mathrm{kg}^{-1} \mathrm{BW}$ GM (Group GM1) on bursal lesions after IBDV infection. In particular, protein expression of IBDV in the bursa decreased in Group GM1, indicating that GM administration decreased IBDV replication in the bursa. Furthermore, immunoglobulin M- and A-bearing B lymphocytes significantly increased 7 days post infection in bursae in Group GM1 $(P<0.01)$, suggesting that the oral administration of $5 \mathrm{mg} \cdot \mathrm{kg}^{-1} \mathrm{GM}$ offers moderate protection against $\mathrm{B}$ cell destruction after IBDV infection. During infection, the concentration of bursal interferon gamma (IFN- $\gamma$ ) increased and peaked in Group GM1 earlier than in Group T (IBDV-exposed), demonstrating that GM administration prompted the production of IFN $-\gamma$ to protect against IBDV infection.
\end{abstract}

Keywords garlic, infectious bursal disease virus (IBDV), antiviral effect, IgM-bearing B lymphocyte

\section{Introduction}

Infectious bursal disease (IBD), caused by the IBD virus (IBDV), is an acute, highly contagious, and immunosuppressive viral disease that infects young chickens ${ }^{[1-4]}$. Because IBDV infection may increase susceptibility to

Received November 5, 2015; accepted December 8, 2015

Correspondences: kedao@cau.edu.cn, yunfeima@cau.edu.cn other pathogens ${ }^{[5]}$ and because of the slow growth rate of infected chickens and their weak responses to subsequent vaccinations $^{[6]}$, IBD causes considerable economic losses in the poultry industry worldwide ${ }^{[7,8]}$. IBDV infects and destroys actively dividing immunoglobulin M-bearing $\left(\operatorname{IgM}^{+}\right)$B lymphocytes in the bursa ${ }^{[9,10]}$. VP2 $(41 \mathrm{kDa})$, a major viral capsid protein of IBDV, confers virulence, cell tropism, and antigenic variation ${ }^{[8,11,12]}$; therefore, VP2 is the obvious target for molecular techniques for IBDV detection $^{[13]}$.

Vaccinations and egg-yolk antibodies are effective preventive and therapeutic options for IBD. Antiviral drug usage has greatly progressed over the past decades; however, some of these drugs may induce viral drug resistance and lead to cell toxicity ${ }^{[14]}$. Garlic (Allium sativum, Liliaceae), a rich source of bioactive compounds, has been safely used as a flavoring, preventive, and curative agent for more than 5000 years worldwide ${ }^{[15,16]}$. It is also notable for its antiarthritic, antimicrobial, antithrombotic, antitumor, hypoglycemic and hypolipidemic properties ${ }^{[17,18]}$. Furthermore, garlic has antiviral activity against several viruses ${ }^{[19]}$. Flavonoids and organosulfur compounds are the two main classes of biological functional components in garlic ${ }^{[20,21]}$. Allicin, which has virucidal activities, is the predominant thiosulfinate in fresh garlic extract ${ }^{[22]}$. Moreover, garlic oil (GO), an oilsoluble garlic extract, is commercially used as a functional food worldwide ${ }^{[23]}$. The major sulfides in GO include diallyl sulfide (57\%), allyl methyl (37\%), and dimethyl sulfide series $(6 \%)^{[15,24]}$. Our previous study has shown that GO plays an active role in resistance against IBDV infection $^{[25]}$; however, GO administration methods are limited because of its solubility. Therefore, we designed a method to extract the water-soluble allicin (garlic mixture, GM) from crushed garlic by steam distillation, but the antiviral effects of GM are little known. Thus, in the study reported here, we examined the effects of oral administration of GM on bursal tissue damage in IBDV-infected specific pathogen-free (SPF) male white leghorn chickens, on changes in bursal $\operatorname{IgM}^{+}$and immunoglobulin A-bearing

(C) The Author(s) 2015. Published by Higher Education Press. This is an open access article under the CC BY license (http://creativecommons.org/licenses/by/4.0) 
$\left(\operatorname{Ig} \mathrm{A}^{+}\right) \mathrm{B}$ lymphocytes, on distribution and load of IBDV in the bursa, and on changes in interferon gamma (IFN- $\gamma$ ) concentrations in the bursal homogenate through histopathological, immunohistochemical, and Western blot analyses, and enzyme-linked immunosorbent assay (ELISA).

\section{Materials and methods}

\subsection{Ethics statement}

The Beijing Municipal Committee of Animal Management and The Ethics Committee of China Agricultural University approved the protocols for animal use and experimentation. All efforts were made to minimize animal suffering.

\subsection{Preparation of GM}

Fresh garlic bulbs (5000 g) were peeled, washed, chopped, and homogenized with distilled water $(25000 \mathrm{~mL})$. After fermentation in a warm bath, the homogenized mixture was subjected to steam distillation twice, and $800 \mathrm{~mL}$ of the distillate was collected. A water solution of hydroxypropyl- $\beta$-cyclodextrin was added to the distillate for preparing the cyclodextrin inclusion compound of allicin. Subsequently, potassium sorbate was dissolved in a suspension of this inclusion compound, which was then diluted to $1 \mathrm{~L}$ with distilled water. Finally, the garlic extract (pH 6.5) was passed through a filter (pore size $0.45 \mu \mathrm{m}$ ), and the allicin content was determined as $1.5 \mathrm{mg} \cdot \mathrm{mL}^{-1}$ using the HPLC method.

\subsection{Animals and viruses}

SPF chickens (Merial Vital Laboratory Animal Technology Co. Ltd, Beijing, China) for the different experimental groups were housed in separate poultry isolators. Feed was provided ad libitum during the experimental period; the amount of water was carefully recorded everyday and provided on demand according to the experimental design. A Chinese virulent strain of the IBDV (BC6/85; CVCC AV7) was obtained from the China Institute of Veterinary Drug Control (Beijing, China). The experimental groups were inoculated with two hundred $50 \%$ mean bird infectious doses of $\mathrm{BC} 6 / 85$ or $0.01 \mathrm{~mol} \cdot \mathrm{L}^{-1}$ phosphate buffered saline (PBS) by eye dropper.

\subsection{Experimental design}

In total, one hundred and twenty 14-day-old SPF chickens were randomized into four groups of 30 chickens each: control (Group C), IBDV-exposed (Group T) and GM administered (Groups GM1 and GM2). When the chickens were 19 days old, GM was added to the water of Groups GM1 (5.0 $\mathrm{mg} \cdot \mathrm{kg}^{-1}$ BW per day) and GM2 $\left(0.2 \mathrm{mg} \cdot \mathrm{kg}^{-1}\right.$ BW per day) for 9 days. Furthermore, when the chickens were 21 days old, Groups T, GM1, and GM2 were inoculated with IBDV BC6/85 by eye dropper. At 1, 2, 3, 4,5 , and 7 days post infection (dpi), five chickens in each group were individually weighed, euthanized by cutting the jugular veins after anesthesia, bled for 3-5 min, and then dissected. Group C was only intraocularly administered sterile PBS and euthanized similarly. Clinical signs of the virus-inoculated chickens were visually examined; bursae from each infected and uninfected chicken were sampled and weighed. The bursal weight index was calculated by dividing bursal weights $(\mathrm{g})$ of chickens and their bodyweights $(\mathrm{kg})$. Moreover, paraffin sections of bursa were prepared for the observation of histopathological lesions by hematoxylin and eosin staining and for the detection of viral antigen, $\operatorname{IgM}^{+}$and $\operatorname{IgA}^{+}$B lymphocytes by immunoperoxidase staining. The bursal homogenate was collected for measuring the IFN- $\gamma$ and IBDV VP2 protein concentration by ELISA and Western blot analysis, respectively.

\subsection{Histopathological examination}

Bursal samples of all groups were fixed in $4 \%$ paraformaldehyde, dehydrated in ascending concentrations of ethyl alcohol, cleaned in benzene, and embedded in melted paraffin wax (P3808; Sigma-Aldrich, St. Louis, MO, USA). The paraffin sections were then cut into $4-\mu \mathrm{m}$-thick slices and stained with hematoxylin and eosin. The stained sections were microscopically observed for assessing histopathological changes.

\subsection{Immunoperoxidase staining}

For immunolabeling of $\operatorname{IgM}^{+} / \operatorname{Ig} \mathrm{A}^{+} \mathrm{B}$ lymphocytes and viral antigens, the paraffin sections were first incubated with one of the following antibodies: goat polyclonal antibody against IgM (A30-102A; Bethyl Laboratories, USA; 1:900) or against IgA (A30-103A; Bethyl Laboratories; 1:300); mouse monoclonal antibody against VP2 (provided by Harbin Veterinary Research Institute, Chinese Academy of Agricultural Sciences, Harbin, China; 1:1000). The sections were incubated with biotinylated rabbit antibody to goat IgG (16-13-06; Kirkegaard and Perry Laboratories, USA; $5 \mu \mathrm{g} \cdot \mathrm{mL}^{-1}$ ) or biotinylated goat antibody to mouse IgG (SP9002; Zhongshan Golden Bridge Biotechnology, Beijing, China) and then with horseradish peroxidase (HRP)-conjugated streptavidin (SP9002; Zhongshan Golden Bridge Biotechnology). Subsequently, the sections were treated with 3,3'-diaminobenzidine-4HCl and $\mathrm{H}_{2} \mathrm{O}_{2}$ (ZLI-9017; Zhongshan Golden Bridge Biotechnology) and counterstained with hematoxylin. 
2.7 Extraction of cytoplasmic proteins, SDS-PAGE and Western blot analysis

At 1, 2, 3, 4, 5, and $7 \mathrm{dpi}$, all bursal samples were frozen in liquid nitrogen and ground into fine powder; $100 \mathrm{mg}$ of each sample was homogenized in $1 \mathrm{~mL}$ cold PBS. The obtained homogenates were mixed with an SDS-PAGE sample loading buffer (P0015; Beyotime Institute of Biotechnology, Jiangsu, China), boiled, treated in an ice bath, and centrifuged. The five treated bursal samples of each group were vortexed, and the supernatant was pooled. The pooled sample was loaded onto slab gels, and the separated proteins were transferred onto a nitrocellulose (NC) membrane. Subsequently, the nitrocellulose membrane was incubated with mouse monoclonal antibody against VP2 (1:1000) or against $\beta$-actin (sc-47778; Santa Cruz Biotechnology Inc., Shanghai, China; 1:2000) and incubated with HRP-conjugated goat antibody to mouse IgG (CW0102; Beijing ComWin Biotech Co. Ltd, Beijing, China; 1:2000). After washing, the NC membrane was incubated with Clarity ${ }^{\mathrm{TM}}$ Western ECL Substrate (1705060; Bio-Rad Laboratories Inc., Hercules, California, USA), and immunoreactive proteins were visualized on an imaging system (VersaDoc; Bio-Rad).

\subsection{Preparation of bursal homogenate and ELISA}

At 2, 3, 4, 5, and $7 \mathrm{dpi}$, all bursal samples were homogenized as described earlier and centrifuged. IFN- $\gamma$ concentrations in the clarified homogenates were measured using an R\&D ELISA kit (R\&D Systems Inc., Minnesota, USA) according to the manufacturer's instructions.

\subsection{Statistical analysis}

Statistically significant differences were analyzed using one-way analysis of variance through multiple comparisons using SPSS Statistics Base 17.0 (SPSS Inc., Chicago, USA). Data were given as mean (M) \pm standard deviation (SD). $P<0.05$ was considered statistically significant.

\section{Results}

After infection with IBDV BC6/85, Groups T and GM1 developed clinical symptoms and characteristic lesions at 2 to $4 \mathrm{dpi}$; these groups gradually recovered from the infection at $5 \mathrm{dpi}$. However, more serious bursal lesions appeared in Group GM2, with severe edema until 7 dpi.

3.1 Effects of GM on the bursal weight index of the IBDVinfected chickens

In particular, the mean bursal indexes of Groups GM1 and GM2 at 1 to 7 and 2 to $7 \mathrm{dpi}$, respectively, were higher than the mean bursal index of Group T. In particular, bursal indexes of Group GM1 at 3 dpi and of GM2 at 3 to 4 dpi were remarkably high $(P<0.05)$. Moreover, bursal indexes of Group $\mathrm{T}$ were significantly lower than those of Group $\mathrm{C}$ at 4 to $7 \mathrm{dpi}(P<0.05)$. However, a significant decrease was observed in Groups GM1 and GM2 at 5 to 7 and $7 \mathrm{dpi}$, respectively $(P<0.05)$ (Fig. 1).

\subsection{Effects of GM on the bursal structure and changes in the B lymphocyte of the IBDV-infected chickens}

A few injured bursal lymphoid follicles persisted in Group $\mathrm{T}$ at $7 \mathrm{dpi}$, and local infiltration of lymphocytes (LIL) and fibrillation were investigated in the interfollicular areas (Fig. 2a, Fig. 2b). Many LIL and a considerable number of original bursal lymphoid follicles were detected, with orderly cortical and medullary epithelial cells and many lymphocytes in the cortex and medulla in Group GM1 at 7 dpi (Fig. 2c, Fig. 2d). In contrast, the most serious pathological changes, such as congestion, edema, hemorrhage and heterophil accumulation, were observed in Group GM2, and a remarkable number of degenerated tissues accumulated in the bursal tube, with few lymphoid follicles observed at 7 dpi (Fig. 2e, Fig. 2f).

Based on these data, the cell types in the bursae of IBDV-infected chickens were further investigated by immunohistochemical analysis. After infection, the number of bursal $\operatorname{IgM}^{+}$B lymphocytes rapidly decreased. Furthermore, at $7 \mathrm{dpi}$, a few $\operatorname{IgM}^{+}$B lymphocytes were scattered in the tissues where lesions involuted in Group T, and LIL were IgM negative (Fig. 3a, Fig. 3b). However, a high number of $\operatorname{IgM}^{+}$B lymphocytes were detected in the cortex and medulla at $7 \mathrm{dpi}$ in Group GM1 (Fig. 3c, Fig. 3d). Also fewer $\operatorname{IgM}^{+}$B lymphocytes were observed up to $7 \mathrm{dpi}$ in the bursal epibiotic area (EA) of Group GM2 (Fig. 3e, Fig. 3f). Similar to the changes found in $\operatorname{IgM}^{+}$B lymphocytes, $\operatorname{IgA}^{+} \mathrm{B}$ lymphocytes were observed in the cortex and medulla after $5 \mathrm{dpi}$ in Group GM1, and LIL were IgA negative (Fig. 3g, Fig. 3h). Few $\operatorname{IgA}^{+} \mathrm{B}$ lymphocytes were observed up to $7 \mathrm{dpi}$ in Groups $\mathrm{T}$ (Fig. 3i, Fig. 3j) and GM2 (Fig. 3k, Fig. 31). At 7 dpi, $\operatorname{IgM}^{+}$ B lymphocytes were enumerated in five fields/bursa/ chicken, and the number of $\operatorname{IgM}^{+}$B lymphocytes in Group GM1 and the epibiotic area in Group GM2 were significantly higher than that in Group $\mathrm{T}(P<0.01)$ (Fig. $3 \mathrm{~m}$ ). The number of $\operatorname{IgA}^{+} \mathrm{B}$ lymphocytes in Group GM1 was significantly higher than that of Group $\mathrm{T}$ at $7 \mathrm{dpi}$ $(P<0.01)$ (Fig. 3n).

3.3 Effects of GM on the expression of bursal viral antigens of the IBDV-infected chickens

After infection, VP2 immunoreactivities revealed an increase-decrease phenomenon, which peaked at 2 to 4 dpi. At 7 dpi, many VP2 immunoreactivities were concentrated in the medulla of injured bursal lymphoid follicles, and some diffusely distributed in the cortex and 


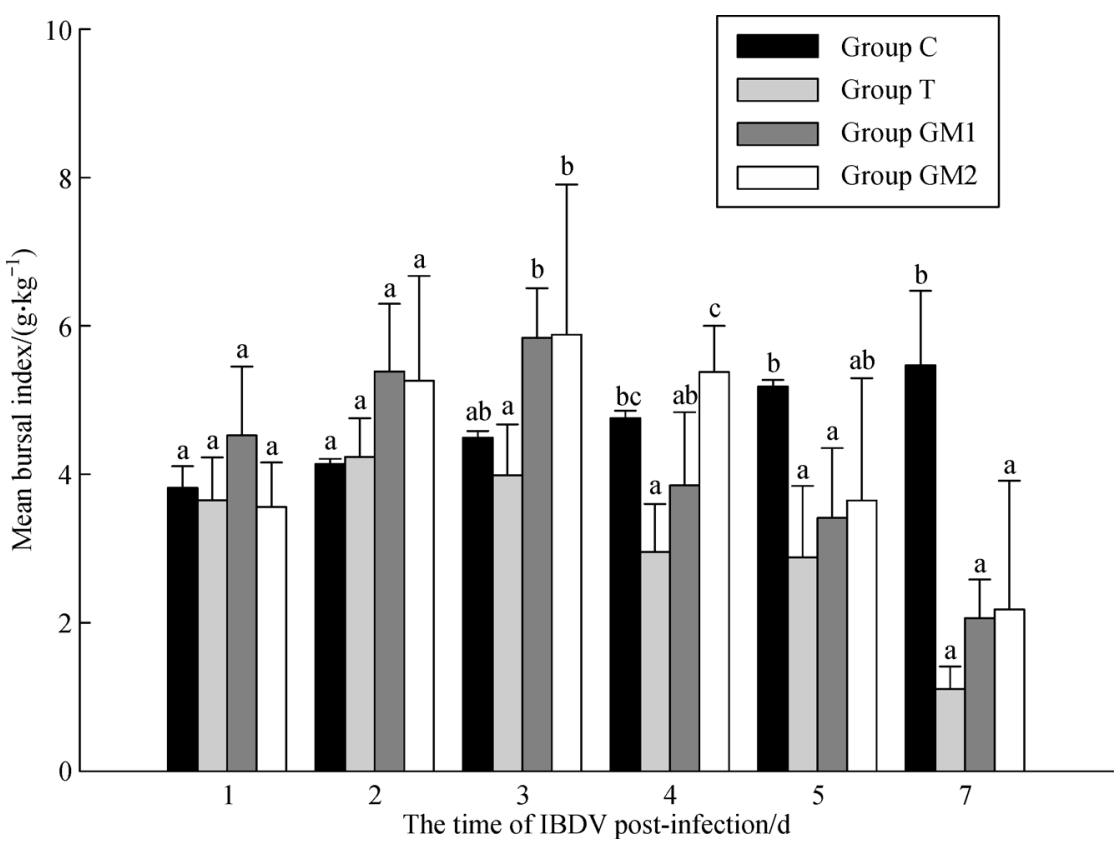

Fig. 1 Effect of GM on the bursal weight index of IBDV-infected chickens. Mean bursal indexes of Groups GM1 and GM2 at 1 to 7 and 2 to 7 dpi, respectively, were higher than the mean bursal index of Group T. Among them, bursal indexes of Groups GM1 and GM2 at 3 and 3 to $4 \mathrm{dpi}$, respectively, were remarkably higher $(P<0.05)$. Bursal indexes of Group T were significantly lower than those of Group $\mathrm{C}$ at 4 to $7 \mathrm{dpi}(P<0.05)$. However, a significant decrease occurred in Groups GM1 and GM2 at 5 to 7 and 7 dpi, respectively $(P<0.05)$. Data were given as mean \pm SD. Data simultaneously marked with different letters indicate significant differences $(P<0.05)$.
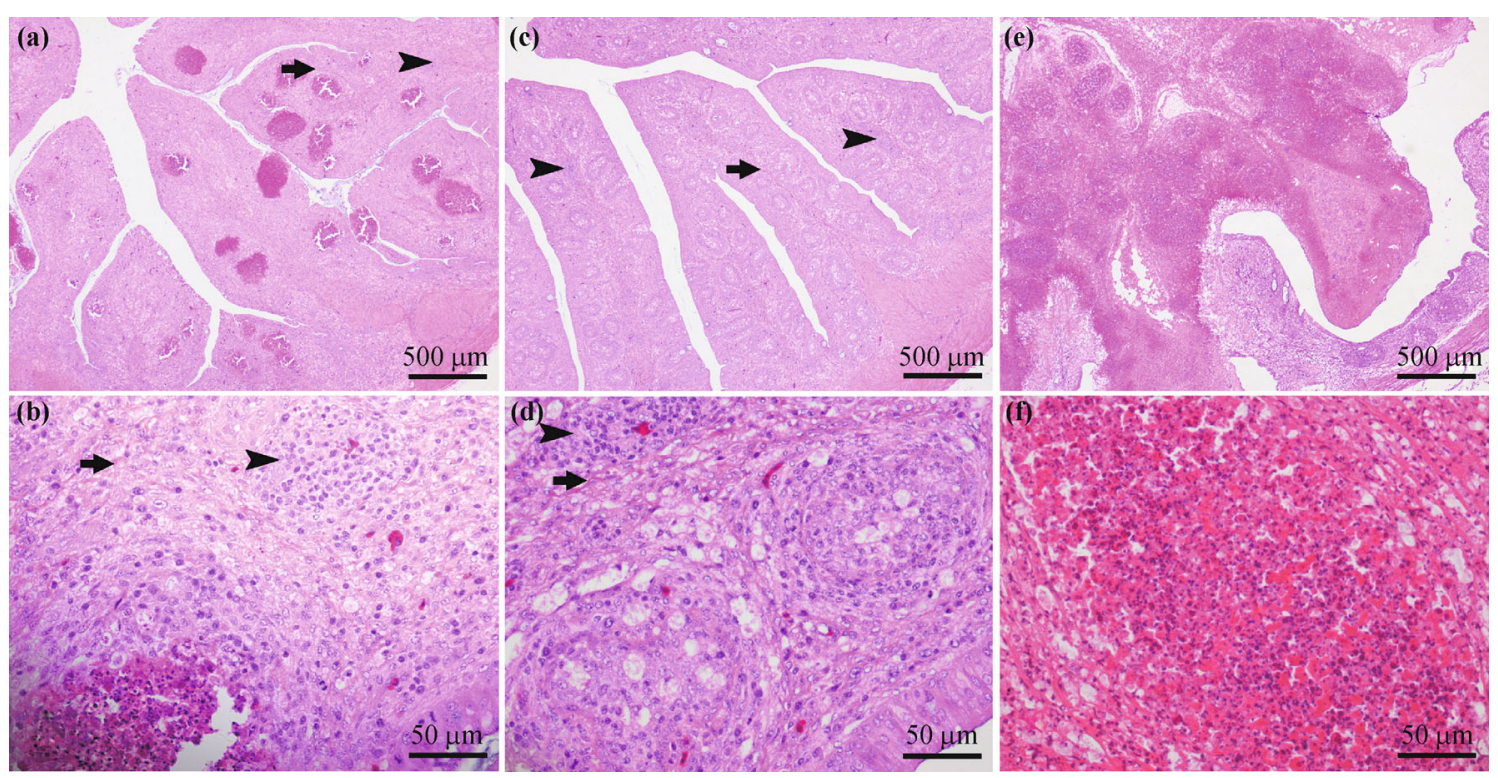

Fig. 2 Effect of GM on the bursal structure changes of IBDV-infected chickens. At 7 dpi, a few injured bursal lymphoid follicles were still observed in Group T, and local infiltration of lymphocytes (LIL) and fibrillation were investigated in the interfollicular areas (a, b). Many LIL and a high number of original bursal lymphoid follicles were detected with orderly cortical and medullary epithelial cells and many lymphocytes in the cortex and medulla in Group GM1 (c, d). The most serious congestion, edema, hemorrhage and heterophil accumulation were observed in Group GM2, and a high number of degenerated tissues accumulated in the bursal tube, with few lymphoid follicles (e, f). Arrows indicate fibrillation. Arrowheads indicate LIL.

interfollicular areas in Group T (Fig. 4a). However, a few VP2 immunoreactivities exhibited diffuse distribution in the plicae in Group GM1 (Fig. 4b). Group GM2 revealed the strongest VP2 immunoreactivities, with most accumu- 

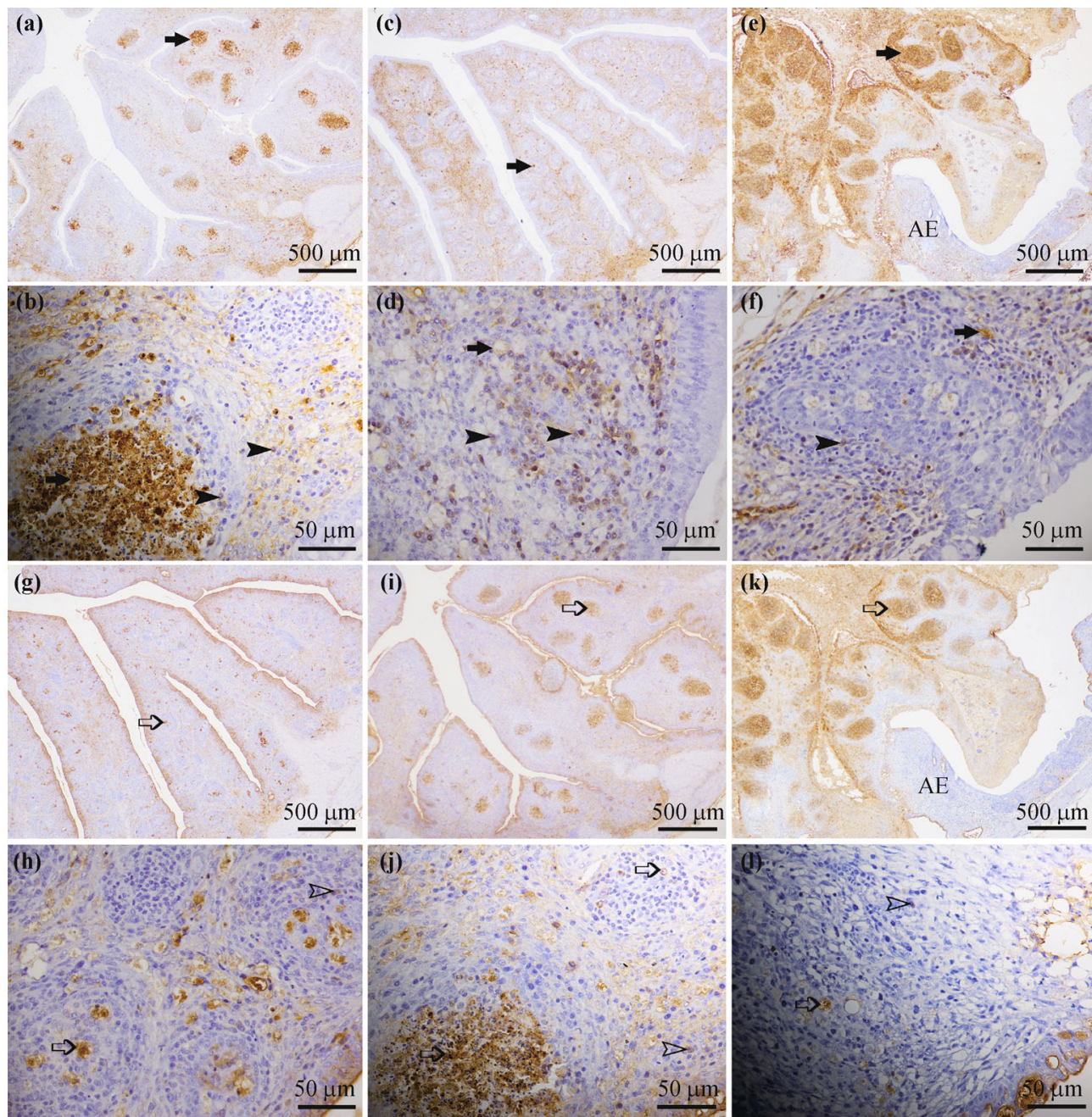

(m)

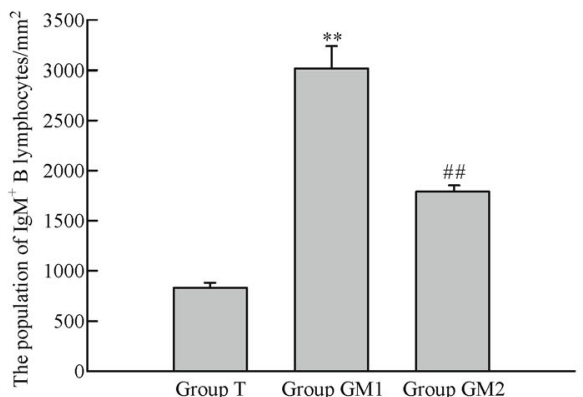

(n)

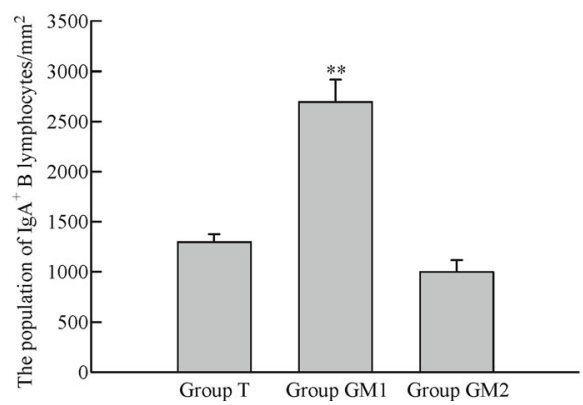

Fig. 3 Effect of GM on the B lymphocyte changes of IBDV-infected chickens. A few $\operatorname{IgM}^{+}$B lymphocytes were scattered in the tissues where lesions involuted in Group T, and LIL were IgM negative $(\mathrm{a}, \mathrm{b})$. A high number of $\operatorname{IgM}^{+} \mathrm{B}$ lymphocytes were detected in the cortex and medulla in Group GM1 (c, d). Fewer IgM ${ }^{+}$B lymphocytes were observed in the bursal epibiotic area (EA) of Group GM2 (e, f). At 7 dpi, many $\operatorname{IgA}^{+}$B lymphocytes were observed in the cortex and medulla in Group GM1, and LIL were $\operatorname{IgA}$ negative $(\mathrm{g}, \mathrm{h})$. Few IgA ${ }^{+}$B lymphocytes were observed in Groups T (i,j) and GM2 (k, 1). At 7 dpi, $\operatorname{IgM}^{+}$B lymphocytes were enumerated in five fields/bursa/ chicken, and a significantly higher number of $\mathrm{IgM}^{+}$B lymphocytes in Group GM1 and the epibiotic area of Group GM2 was observed than in Group T $(P<0.01)(\mathrm{m})$. The number of $\operatorname{IgA}^{+}$B lymphocytes in Group GM1 was significantly higher than that in Group $\mathrm{T}$ at $7 \mathrm{dpi}$ $(P<0.01)(\mathrm{n})$. Solid arrows and arrowheads indicate scattered IgM immunoreactivities and $\operatorname{IgM}^{+}$B lymphocytes, respectively. Feint arrows and arrowheads indicate scattered $\operatorname{IgA}$ immunoreactivities and $\operatorname{IgA}^{+} \mathrm{B}$ lymphocytes, respectively. Data are given as mean $\pm \mathrm{SD}$. **, $P<0.01$ between Groups GM1 and T; ${ }^{\# \#}, P<0.01$ between Groups GM2 and T. 
lated in the degenerated tissues (Fig. 4c). Western blot analysis (Fig. 4d, Fig. 4e) confirmed the low VP2 expression in Group GM1.

3.4 Effects of GM on IFN- $\gamma$ concentrations in the bursal homogenate of the IBDV-infected chickens

After infection, bursal IFN- $\gamma$ concentrations increased in the chickens. IFN- $\gamma$ concentrations in Groups T and GM1 initially increased and subsequently decreased, with a significant increase at 4 and $5 \mathrm{dpi}$ in Group T and 2 to $4 \mathrm{dpi}$ in Group GM1 $(P<0.05)$. A significantly high bursal IFN$\gamma$ concentration was observed in Group GM2 at 4 and 7 dpi $(P<0.05)$. The IFN- $\gamma$ concentration peaked at 3 dpi in Group GM1, 2 days earlier than in Groups T and GM2 (Fig. 5).

\section{Discussion}

IBD is an economically important, immunosuppressionrelated infectious disease of young chickens worldwide ${ }^{[26]}$. BC6/85 is a standard virulent IBDV strain and is widely used in China for evaluating the efficacy of IBDV vaccines ${ }^{[13]}$. The present study confirmed our previous reports that 3-week-old chickens infected with IBDV BC6/ 85 exhibit clinical symptoms and characteristic pathological changes ${ }^{[13,27]}$. Therefore, the above model is suitable for assessing the preventive and therapeutic effects of GM.

IBDV infection severely depletes the B lymphocytes and destroys bursal tissues, in addition to severely depleting the medulla of the follicles ${ }^{[27-30]}$. In the present study, a high number of lymphocytes were detected in the lymphoid follicles in Group GM1 at 5 to $7 \mathrm{dpi}$; therefore, we speculated that a number of B lymphocytes were preserved because of the oral administration of $5 \mathrm{mg} \cdot \mathrm{kg}^{-1} \mathrm{GM}$. Further research revealed a markedly higher number of $\operatorname{IgM}^{+}$and $\operatorname{IgA}^{+}$B lymphocytes in Group GM1 than in Group $\mathrm{T}$, suggesting that the higher bursal weight indexes in Group GM1 were associated with the high number of preserved $\mathrm{IgM}^{+}$and $\mathrm{IgA}^{+}$B lymphocytes. Our data suggest that the oral administration of $5 \mathrm{mg} \cdot \mathrm{kg}^{-1} \mathrm{GM}$ confers moderate protection against $\mathrm{B}$ cell destruction after IBDV infection. Similar to the present findings, Salman et al. and Milner reported that garlic derivatives activate stimulatory properties in lymphocytes ${ }^{[17,31]}$.

Garlic is favored for its antiviral properties, and our previous research indicated that the oral administration of $10 \mathrm{mg} \cdot \mathrm{kg}^{-1} \mathrm{GO}$ decreases IBDV replication ${ }^{[25]}$. Therefore, we investigated IBDV replication and distribution in the bursa. Western blot analyses and immunoperoxidase staining exhibited low IBDV VP2 expression in Group GM1, indicating the anti-IBDV effect of oral administration of $5 \mathrm{mg} \cdot \mathrm{kg}^{-1} \mathrm{GM}$. Kim et al. indicated that IFN- $\gamma$ is crucial in inducing antiviral effects by immune cells such as macrophages and cytotoxic $\mathrm{T}$ lymphocytes ${ }^{[32]}$; these results are consistent with our results: bursal IFN- $\gamma$ concentration increased in the IBDV-infected chickens, and that in Group GM1 peaked at 3 dpi, 2 days earlier than the peak observed in Groups T and GM2. Therefore, we assume that IBDV infection stimulates IFN- $\gamma$ production and that the oral administration of $5 \mathrm{mg} \cdot \mathrm{kg}^{-1} \mathrm{GM}$

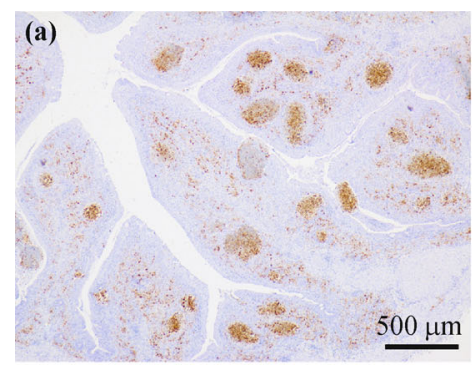

(b)
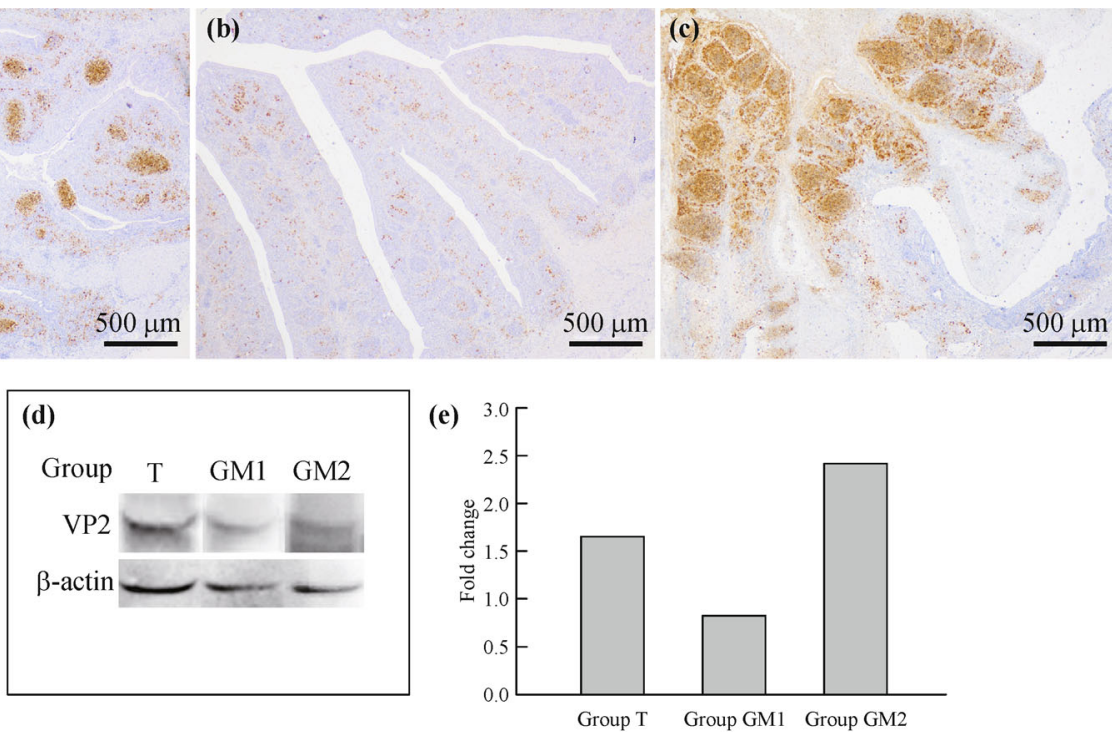

Fig. 4 Effect of GM on the expression of bursal viral antigens of IBDV-infected chickens. At 7 dpi, many VP2 immunoreactivities were concentrated in the medulla of injured bursal lymphoid follicles, and some diffusely distributed in the cortex and interfollicular areas in Group T (a). A few VP2 immunoreactivities exhibited diffuse distribution in the plicae in Group GM1 (b). The strongest VP2 immunoreactivities were observed in Group GM2, with most accumulated in degenerated tissues (c). Western blot analysis (d, e) confirmed the low VP2 expression in GM1. 


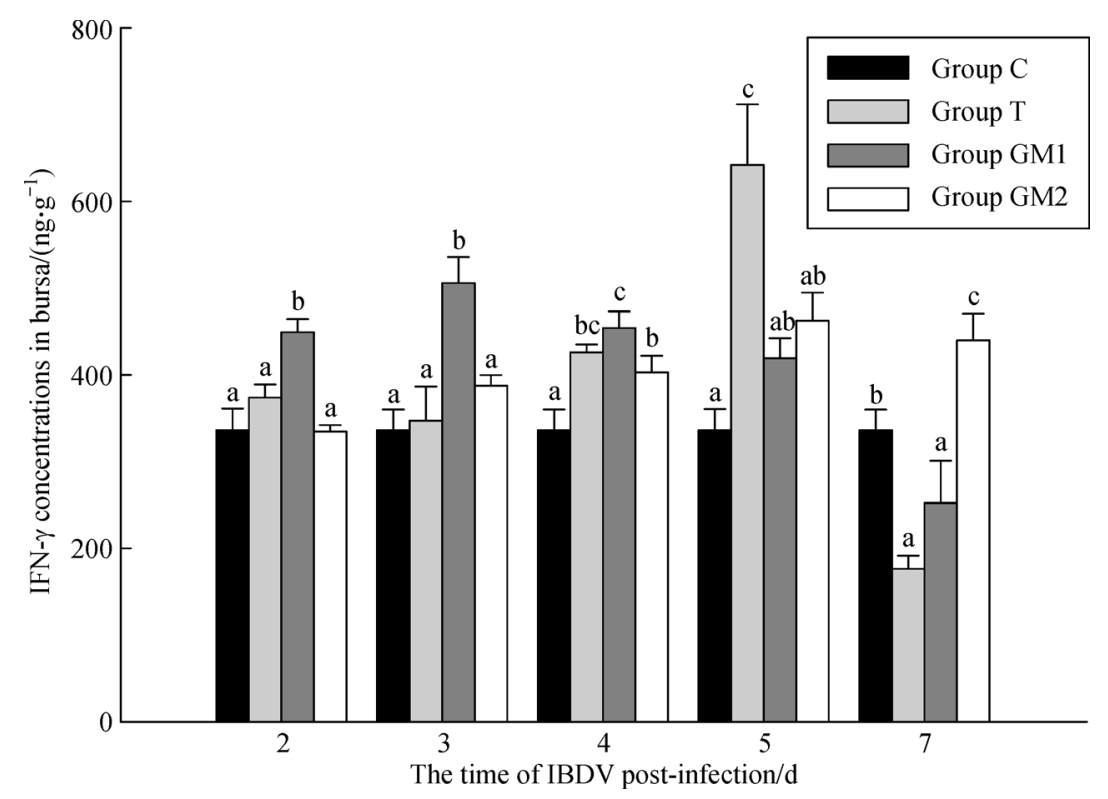

Fig. 5 Effect of GM on IFN- $\gamma$ concentrations in the bursal homogenate of IBDV-infected chickens. After infection, bursal IFN- $\gamma$ concentrations increased in the chickens. Among them, IFN- $\gamma$ concentrations in Groups T and GM1 initially increased and subsequently decreased, with a significant increase at 4 and 5 dpi in Group T and 2 to 4 dpi in Group GM1 $(P<0.05)$. A significantly higher bursal IFN- $\gamma$ concentration was observed in Group GM2 at 4 and 7 dpi $(P<0.05)$. The IFN- $\gamma$ concentration peaked at 3 dpi in Group GM1, 2 days earlier than that in Groups T and GM2. Data are given as mean \pm SD. Data simultaneously marked with different letters indicate significant differences $(P<0.05)$.

strengthens the stimulating effects. T lymphocytes were first detected at $4 \mathrm{dpi}$; $65 \%$ of bursal cells are $\mathrm{T}$ lymphocytes at $7 \mathrm{dpi}^{[32]}$, and these lymphocytes play a substantial role in conferring resistance against IBDV ${ }^{[33]}$. Furthermore, our data revealed that LIL are neither $\operatorname{IgM}^{+}$ nor $\mathrm{IgA}^{+} \mathrm{B}$ lymphocytes, which were the most likely $\mathrm{T}$ lymphocytes and were observed more frequently in Group GM1. Similar to our findings, Kim et al. reported Tlymphocyte infiltration in IBDV-infected bursa using immunohistochemical analysis ${ }^{[32]}$. Moreover, IBDV infection recruited activated macrophages into the bursa ${ }^{[27]}$. Thus, local infiltration of $\mathrm{T}$ lymphocytes and macrophage activation may confer antiviral activities through high IFN- $\gamma$ concentrations.

In the present study, IBDV infection caused bursal lesions in Groups T, GM1, and GM2. A higher bursal viral load and serious pathological lesions were observed in Group GM2 because the administered concentration of GM $\left(0.2 \mathrm{mg} \cdot \mathrm{kg}^{-1}\right)$ possibly did not reach the minimal effective antiviral dose. Adverse effects of garlic, such as abdominal pain, appetite loss, bloating and bad breath, are well known; an excessive intake of garlic may also adversely affect the liver and muscle cells ${ }^{[19]}$. Our pilot studies have revealed that the oral administration of $10 \mathrm{mg} \cdot \mathrm{kg}^{-1} \mathrm{GM}$ for 4 days causes vent pecking in $60 \%$ chickens (data not shown). Therefore, we speculate the presence of some other pungent components in GM. Further studies must be conducted for optimizing a simple and low-cost extraction method for GM.

\section{Conclusions}

These results show that the oral administration of $5 \mathrm{mg} \cdot \mathrm{kg}^{-1} \mathrm{GM}$ regulates the secretion of bursal IFN- $\gamma$, decreases bursal viral load, and protects the bursal structure and lymphocytes against IBDV infection. In conclusion, GM has an antiviral activity against IBDV.

Acknowledgements The authors wish to thank Prof. Jianqin $\mathrm{Xu}$ and Fenghua Liu for their expert technical assistance in preparation of GM. This work was supported by the Twelfth Five-Year-Plan of the National Science and Technology Support Project (2011BAD34B01) and National Natural Science Foundation of China (31502025).

Compliance with ethics guidelines Sufen Zhao, Yuanyuan Jia, Weiwei Zhang, Lili Wang, Yunfei Ma, and Kedao Teng declare that they have no conflict of interest or financial conflicts to disclose.

All applicable institutional and national guidelines for the care and use of animals were followed.

\section{References}

1. Abdel-Moneim A S, Abdel-Gawad M M. Genetic variations in maternal transfer and immune responsiveness to infectious bursal disease virus. Veterinary Microbiology, 2006, 114(1-2): 16-24

2. Khatri M, Palmquist J M, Cha R M, Sharma J M. Infection and activation of bursal macrophages by virulent infectious bursal disease virus. Virus Research, 2005, 113(1): 44-50

3. Petek M, D'Aprile P N, Cancellotti F. Biological and physicochemical properties of the infectious bursal disease virus (IBDV). 
Avian Pathology, 1973, 2(2): 135-152

4. Stoute S T, Jackwood D J, Sommer-Wagner S E, Crossley B M, Woolcock P R, Charlton B R. Pathogenicity associated with coinfection with very virulent infectious bursal disease and Infectious bursal disease virus strains endemic in the United States. Journal of Veterinary Diagnostic Investigation, 2013, 25(3): 352358

5. Li Z, Wang Y, Li X, Cao H, Zheng S J. Critical roles of glucocorticoid-induced leucine zipper in infectious bursal disease virus (IBDV)-induced suppression of type I Interferon expression and enhancement of IBDV growth in host cells via interaction with VP4. Journal of Virology, 2013, 87(2): 1221-1231

6. Müller H, Islam M R, Raue R. Research on infectious bursal disease-the past, the present and the future. Veterinary Microbiology, 2003, 97(1-2): 153-165

7. Liang J F, Yin Y Y, Qin T, Yang Q. Chicken bone marrow-derived dendritic cells maturation in response to infectious bursal disease virus. Veterinary Immunology and Immunopathology, 2015, 164(12): $51-55$

8. Stricker R L, Behrens S E, Mundt E. Nuclear factor NF45 interacts with viral proteins of infectious bursal disease virus and inhibits viral replication. Journal of Virology, 2010, 84(20): 1059210605

9. Hirai K, Funakoshi T, Nakai T, Shimakura S. Sequential changes in the number of surface immunoglobulin-bearing B lymphocytes in infectious bursal disease virus-infected chickens. Avian Diseases, 1981, 25(2): 484-496

10. Rodenberg J, Sharma J M, Belzer S W, Nordgren R M, Naqi S. Flow cytometric analysis of $\mathrm{B}$ cell and $\mathrm{T}$ cell subpopulations in specificpathogen-free chickens infected with infectious bursal disease virus. Avian Diseases, 1994, 38(1): 16-21

11. Mundt E, Beyer J, Müller H. Identification of a novel viral protein in infectious bursal disease virus-infected cells. Journal of General Virology, 1995, 76(2): 437-443

12. Xu H, Yuan L, Wang F, Wang Y, Wang R, Song C, Xia Q, Zhao P. Overexpression of recombinant infectious bursal disease virus (IBDV) capsid protein VP2 in the middle silk gland of transgenic silkworm. Transgenic Research, 2014, 23(5): 809-816

13. Wang A R, Liu F H, Wang Z P, Jiang X, Wang W, Teng K D, Xu J. Pathological study of SPF chickens experimentally infected with a Chinese IBDV strain BC6/85. Asian Journal of Animal and Veterinary Advances, 2011, 6(1): 36-50

14. Li Y, Wang L, Li S, Chen X, Shen Y, Zhang Z, He H, Xu W, Shu Y, Liang G, Fang R, Hao X. Seco-pregnane steroids target the subgenomic RNA of alphavirus-like RNA viruses. Proceedings of the National Academy of Sciences of the United States of America, 2008, 104(19): 8083-8088

15. Amagase H. Significance of garlic and its constituents in cancer and cardiovascular disease. Clarifying the real bioactive constituents of garlic. Journal of Nutrition, 2006, 136: 716S-725S

16. Boonpeng S, Siripongvutikorn S, Sae-Wong C, Sutthirak P. The antioxidant and anti-cadmium toxicity properties of garlic extracts. Food Science \& Nutrition, 2014, 2(6): 792-801

17. Milner J A. Significance of garlic and its constituents in cancer and cardiovascular disease. Preclinical perspectives on garlic and cancer.
Journal of Nutrition, 2006, 136: 827S-831S

18. Thomas S, Senthilkumar G P, Sivaraman K, Bobby Z, Paneerselvam $\mathrm{S}$, Harichandrakumar $\mathrm{K} \mathrm{T}$. Effect of s-methyl-L-cysteine on oxidative stress, inflammation and insulin resistance in male wistar rats fed with high fructose diet. Iranian Journal of Basic Medical Sciences, 2015, 40(1): 45-50

19. Majewski M. Allium sativum: facts and myths regarding human health. Roczniki Panstwowego Zakladu Higieny, 2014, 65(1): 18

20. Asadpour R, Azari M, Hejazi M, Tayefi H, Zaboli N. Protective effects of garlic aquous extract (Allium sativum), vitamin $\mathrm{E}$, and $\mathrm{N}-$ acetylcysteine on reproductive quality of male rats exposed to lead. Veterinary Research Forum : An International Quarterly Journal, 2013, 4(4): 251-257

21. Miron T, Rabinkov A, Mirelman D, Wilchek M, Weiner L. The mode of action of allicin: its ready permeability through phospholipid membranes may contribute to its biological activity. Biochimica et Biophysica Acta, 2000, 1463(1): 20-30

22. Weber N D, Andersen D O, North J A, Murray B K, Lawson L D, Hughes B G. In vitro virucidal effects of Allium sativum (garlic) extract and compounds. Planta Medica, 1992, 58(5): 417-423

23. Zeng T, Zhang C L, Song F Y, Han X Y, Xie K Q. The modulatory effects of garlic oil on hepatic cytochrome P450s in mice. Human and Experimental Toxicology, 2009, 28(12): 777-783

24. Lawson L D, Wang Z J, Hughes B G. Identification and HPLC quantitation of the sulfides and $\operatorname{dialk}(\mathrm{en}) \mathrm{yl}$ thiosulfinates in commercial garlic products. Planta Medica, 1991, 57(04): 363-370

25. Wang A R. Development of an experimental model of IBDV infection and a preliminary study for antiviral action of garlic oil. Dissertation for the Doctoral Degree. Beijing: China Agriculture University, 2009 (in Chinese)

26. Maity H K, Dey S, Mohan C M, Khulape S A, Pathak D C, Vakharia V N. Protective efficacy of a DNA vaccine construct encoding the VP2 gene of infectious bursal disease and a truncated HSP70 of Mycobacterium tuberculosis in chickens. Vaccine, 2015, 33(8): 1033-1039

27. Ma H, Zhao S, Ma Y, Guo X, Han D, Jia Y, Zhang W, Teng K. Susceptibility of Kupffer cells to virus in chickens experimentally infected with Chinese virulent IBDV. Veterinary Microbiology, 2013, 164(3-4): 270-280

28. Bíró E, Kocsis K, Nagy N, Molnár D, Kabell S, Palya V, Oláh I. Origin of the chicken splenic reticular cells influences the effect of the infectious bursal disease virus on the extracellular matrix. Avian Pathology, 2011, 40(2): 199-206

29. Dobrosavljević I, Vidanović D, Velhne M, Miljkovi Bć B, Lako. Simultaneous detection of vaccinal and field infectious bursal disease viruses in layer chickens challenged with a very virulent strain after vaccination. Acta Veterinaria Hungarica, 2014, 62(2): 264-273

30. Käufer I, Weiss E. Significance of bursa of Fabricius as target organ in infectious bursal disease of chickens. Infection and Immunity, 1980, 27: 364-367

31. Salman H, Bergman M, Bessler H, Punsky I, Djaldetti M. Effect of a garlic derivative (alliin) on peripheral blood cell immune responses. International Journal of Immunopharmacology, 1999, 21(9): 589- 
597

32. Kim I J, You S K, Kim H, Yeh H Y, Sharma J M. Characteristics of bursal $\mathrm{T}$ lymphocytes induced by infectious bursal disease virus. Journal of Virology, 2000, 74(19): 8884-8892
33. Rautenschlein S, Yeh H Y, Sharma J M. The role of T cells in protection by an inactivated infectious bursal disease virus vaccine. Veterinary Immunology and Immunopathology, 2002, 89(3-4): 159-167 